\title{
Surgical options in infective valve endocarditis with neurological complications
}

\author{
Khalil Jawad $^{1,2 \#}$, Guenther Kroeg ${ }^{1 \#}$, Alex Koziarz ${ }^{2}$, Sven Lehmann ${ }^{1}$, Maja Dieterlen ${ }^{1}$, Stefan Feder ${ }^{1}$, \\ Jens Garbade ${ }^{1}$, Vivek Rao ${ }^{2}$, Michael Borger ${ }^{1}$, Martin Misfeld $^{1}$ \\ ${ }^{1}$ Department of Cardiac Surgery, University of Leipzig, Heart Center Leipzig, Helios Clinic, Leipzig, Germany; ${ }^{2}$ Toronto General Hospital, \\ University of Toronto, Toronto, Ontario, Canada \\ \#These authors contributed equally to this work. \\ Correspondence to: Khalil Jawad. Department of Cardiac Surgery, Heart Center, Leipzig University, Struempellstrasse 39, 04289 Leipzig, Germany. \\ Email: khalil.jawad@medizin.uni-leipzig.de.
}

\begin{abstract}
Background: Surgery is a common treatment option for patients with infective endocarditis. We present a large cohort of surgically managed patients with infective endocarditis and evaluate the long-term mortality of those with and without neurological complications.

Methods: We performed a retrospective review of patients surgically managed for infective endocarditis between 1994 and 2017. Demographic and outcome data were collected using a hospital database. Time-toevent analysis was performed with Kaplan-Meier curve and compared statistically with log-rank testing.

Results: At the time of admission, 680 (27.7\%) patients with infective endocarditis showed neurological complications. The mean age was $62.6 \pm 14.0$ years and $70 \%$ were male. Two thousand two hundred and sixty-one (92\%) patients had left-sided valve endocarditis. Isolated aortic valve endocarditis was present in $59 \%$ of patients and $35 \%$ of patients had isolated infective endocarditis of the mitral valve. Mean logistic EuroSCORE was 21.2 \pm 21.6 . Microbiologic cultures were positive in 1,939 patients (79\%). The most common bacteria were Staphylococcus aureus (26\%). In-hospital mortality in the group of patients with Staphylococcus aureus was significantly higher than in the group infected with other pathogens $(18.2 \%$ vs. $13.4 \%, \mathrm{P}=0.004)$. Patients with vegetations $\geq 1 \mathrm{~cm}$ had significantly more systemic embolization $(\mathrm{P}<0.001)$. $44 \%$ of patients had septic embolization with the most common site being the spleen, followed by the brain. Patients presenting with neurological complications had significantly higher in-hospital and long-term mortality $(\mathrm{P}<0.0001)$.

Conclusions: Infective endocarditis patients with neurological complications have a significantly higher risk of mortality than patients without neurological complications. Surgery is an effective treatment in patients presenting with infective endocarditis, and may be undertaken in patients with neurological complications to prevent poorer prognosis.
\end{abstract}

Keywords: Valve endocarditis; neurological complication; infectious disease

Submitted Aug 16, 2019. Accepted for publication Oct 12, 2019.

doi: $10.21037 /$ acs.2019.10.04

View this article at: http://dx.doi.org/10.21037/acs.2019.10.04

\section{Introduction}

Infective endocarditis (IE) is an infectious disease associated with high morbidity and mortality. It is one of the most common life-threatening infections, occurring more frequently in older patients and those with prosthetic valves (1). Due to severe complications in these patients, surgical treatment is required in approximately half of cases (2-4). Symptomatic neurological complications occur in 15$30 \%$ of patients with infective endocarditis (2). The clinical outcome is associated with a poor prognosis (5). Common neurological complications include transient ischemic 
attack, intracerebral hemorrhage, brain abscess, and toxic encephalopathy (3). However, silent cerebral embolisms also occur and may be associated with poorer prognosis (5). Surgical treatment is particularly effective in select IE patients presenting with neurological complications to prevent neurological sequelae and mortality. However, the decision for surgery remains controversial and should be decided carefully with a multidisciplinary team as patient prognosis may worsen with surgical intervention. Anticoagulation during cardiopulmonary bypass may be particularly harmful for some patients (5). However, some studies have shown that neurological complications are not a contraindication for emergency surgery (6). The optimal time interval between neurological complications and surgery is controversial, however, recent guidelines favor surgery in cases presenting with heart failure, uncontrolled infection, abscess formation, and those with high embolic risk $(3,7)$. In this article, we evaluate a large cohort of IE patients and compare the mortality of patients presenting with and without neurological complications.

\section{Methods}

We retrospectively reviewed our database for patients who underwent surgical treatment for IE between 1994 and 2017. All our clinical data were obtained from patient records and our institutional database. Modified Duke criteria was used to diagnose IE (8) and a standardized protocol was used for all patients admitted to hospital with IE. Transthoracic or transesophageal echocardiography was done to measure and classify vegetations. Blood cultures and full body computed tomography scans were routinely done. Once the diagnosis was confirmed, empiric antibiotic therapy was started immediately. Patients' indication for surgery was according to the ESC/EACTS guidelines for the management of IE (3). The local ethics committee did not require additional individual patient consent.

All patients suspected by a clinician to have neurological complications were evaluated by a neurologist. Patients with neurological deficit were considered as symptomatic and included in the neurological complications group. Asymptomatic patients with or without neurological abnormalities on MRI or CT were included in the nonneurological complications group. Follow-up data were acquired by reviewing the prospective hospital database results and reviewing follow-up information obtained by annual written or telephone interviews. Continuous variables were presented as mean and standard deviation.
Categorical variables were presented as counts with percentages. Kaplan-Meier actuarial analyses were performed with the Greenwood formula for variance. A $\mathrm{P}$ value less than 0.05 was considered as statistically significant. Statistical analyses were performed using IBM SPSS Statistics, version 24 (SPSS Inc, Chicago, IL).

\section{Results}

Between 1994 and 2017, 2,568 patients received open heart surgery for IE in our center. One hundred and ten patients were excluded due to missing data, yielding a total of 2,458 patients for final inclusion. Follow-up data was $100 \%$ complete for included patients and ranged from 0 to 15 years. At the time of admission, $26.5 \%$ of patients showed neurological complications. The mean age was $62.6 \pm 14.0$ years and $1,721(70 \%)$ were male (Table 1$)$. The mean left ventricular ejection fraction (LVEF) was $55 \%$, with one third of cases having LVEF $<50 \%$ and approximately $10 \%$ of cases having LVEF $<30 \%$. Leftsided IE was predominant affecting $92 \%$ of patients. Mean logistic EuroSCORE was $21.2 \pm 21$.6. Mean body mass index was $26.74 \pm 5 \mathrm{~kg} / \mathrm{m}^{2}$. A total of 651 patients (26\%) had coronary artery disease. The majority of patients presented with comorbidities, which included diabetes $(32 \%)$, peripheral vascular disease $(11 \%)$, arterial hypertension (65\%), hyperlipidemia (30\%), and chronic obstructive pulmonary disease $(11 \%)$. Isolated aortic valve endocarditis was present in $59 \%$ of patients, followed by $35 \%$ for isolated mitral valve, $5 \%$ isolated tricuspid valve, and $1 \%$ isolated pulmonary valve (Figure 1 ). The most common mode of valve function deterioration was mitral insufficiency, followed by aortic insufficiency, tricuspid insufficiency, and aortic stenosis (Figure 2).

In $17 \%$ of patients, preoperative blood cultures were negative. Microbiologic cultures were positive in 1,939 (79\%) patients. The original infection was determined in $51 \%$ of patients with the most common origin being dermatological, followed by pulmonary, urological, intestinal, and dental (Figure 3). The most common bacteria were Staphylococcus aureus (26\%), followed by streptococcus spp. (21\%) Figure 4. The mean size of the valve vegetation was $8 \mathrm{~mm} .63 \%$ of vegetations assessed with echocardiography measured $\geq 1 \mathrm{~cm}$. Six patients $(0.24 \%)$ had a vegetation larger than $3 \mathrm{~cm}$. Patients with vegetations $\geq 1 \mathrm{~cm}$ had significantly more systemic embolization than patients with vegetations $<1 \mathrm{~cm}(\mathrm{P}<0.001)$. In our cohort, $44 \%$ of patients showed a septic embolization with the most 
Table 1 Demographics of 2,458 included patients with infective endocarditis

\begin{tabular}{|c|c|}
\hline Demographics & $\mathrm{N}[\%]$ \\
\hline Age (years $\pm \mathrm{SD}$ ) & $62.6 \pm 14$ \\
\hline Women & $732[30]$ \\
\hline $\mathrm{BMI}\left(\mathrm{kg} / \mathrm{m}^{2}, \pm \mathrm{SD}\right)$ & $26.74 \pm 5$ \\
\hline Arterial hypertension & $1,606[65]$ \\
\hline Diabetes mellitus 2 & 795 [32] \\
\hline Hypercholesterolemia & 737 [30] \\
\hline Renal insufficiency & 858 [35] \\
\hline Dialysis & $160[7]$ \\
\hline PAD & $268[11]$ \\
\hline COPD & $277[11]$ \\
\hline CAD any stenosis & $651[26]$ \\
\hline Septic shock & $247[10]$ \\
\hline NYHA Class III or IV & $1,251[51]$ \\
\hline Reoperation & 790 [32] \\
\hline Logistic ES (\% \pm SD) & $21.2 \pm 21.6$ \\
\hline Mean LVEF & 55 \\
\hline
\end{tabular}

$\mathrm{BMI}$, body mass index; PAD, peripheral artery disease; COPD, chronic obstructive pulmonary disease; CAD, coronary artery disease; NYHA, New York Heart Association; ES, EuroSCORE; LVEF, left ventricular ejection fraction. Categorical variables are provided as counts with percentages. Continuous variables are provided as mean with standard deviation.

common site being the spleen, followed by brain, kidney, extremities, and lung (Figure 5). 26\% of patients had preoperative symptomatic neurological dysfunction which was significantly associated with the brain embolization $(\mathrm{P}<0.001$; Figure 6).

The in-hospital mortality in patients with Staphylococcus aureus cultures was significantly higher than in the group affected by other pathogens ( $18.2 \%$ vs. $13.4 \%, \mathrm{P}=0.004)$. Concomitant procedures were performed in $44 \%$ of the cases including coronary artery bypass grafting in 407 patients $(16.5 \%)$, mitral valve repair or replacement in $46.4 \%$, and replacement of the aortic root in $23.4 \%$. No aortic root enlargement procedures were performed. The mean cross-clamp time was $85.3 \pm 44.1$ minutes, and mean cardiopulmonary bypass time was $126.3 \pm 67.5$ minutes. Rethoracotomy due to bleeding was necessary in $333(13.55 \%)$

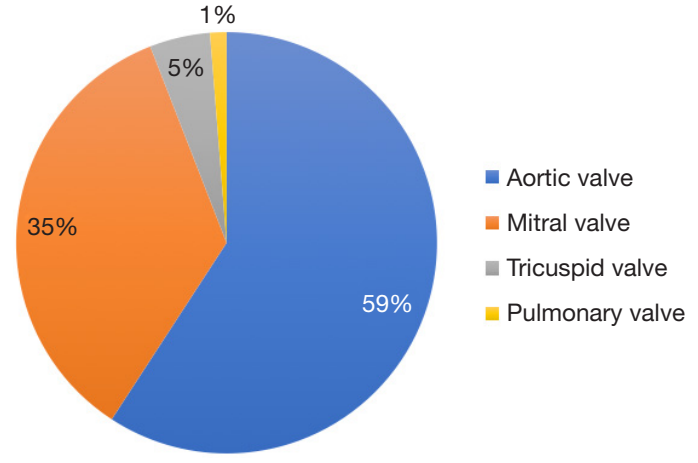

Figure 1 Distribution of isolated infected valves ( $N=1,980$ patients).

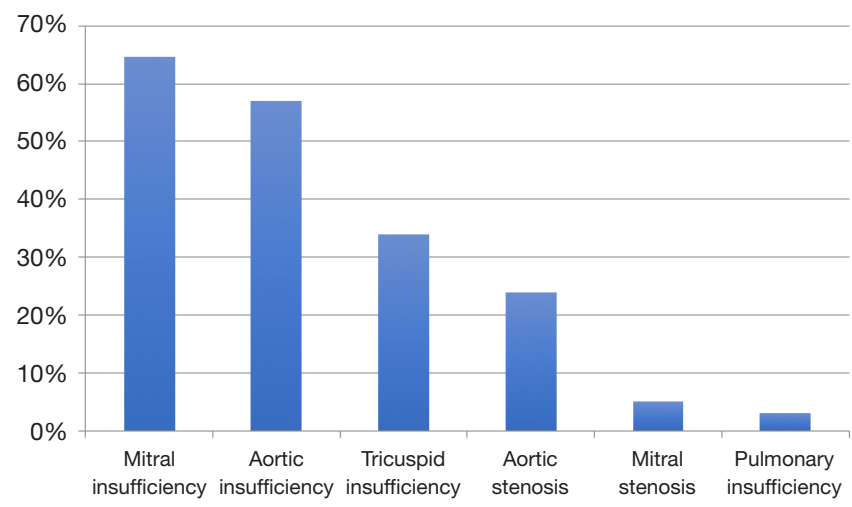

Figure 2 Distribution of valve failure.

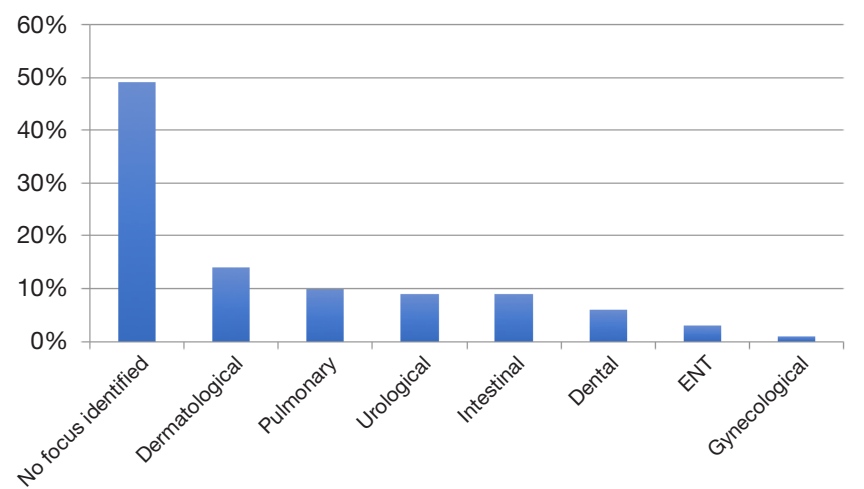

Figure 3 Infection focus. ENT indicates ear, nose or throat focus.

patients.

The most frequently observed complications included cerebrovascular accident in 645 patients $(26.2 \%)$. Respiratory failure occurred in 328 (13.3\%) patients, and acute renal failure requiring temporary renal replacement therapy occurred in $512(20.8 \%)$ patients. The 30-day 

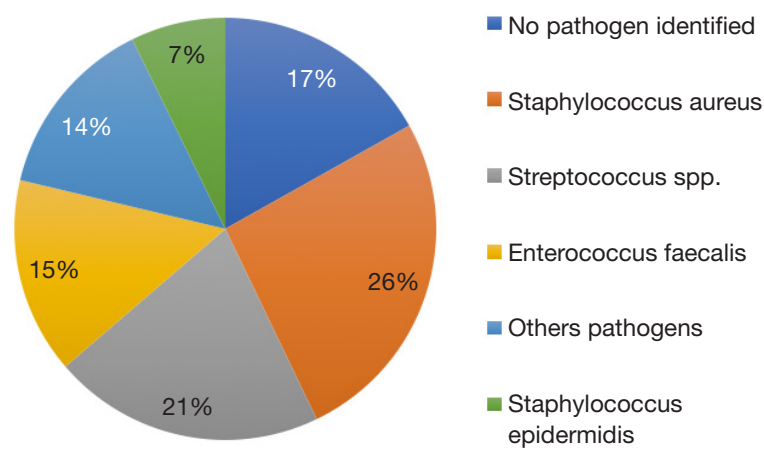

Figure 4 Microbiological pathogen identification.



Figure 5 Frequency of septic embolization by target organ.

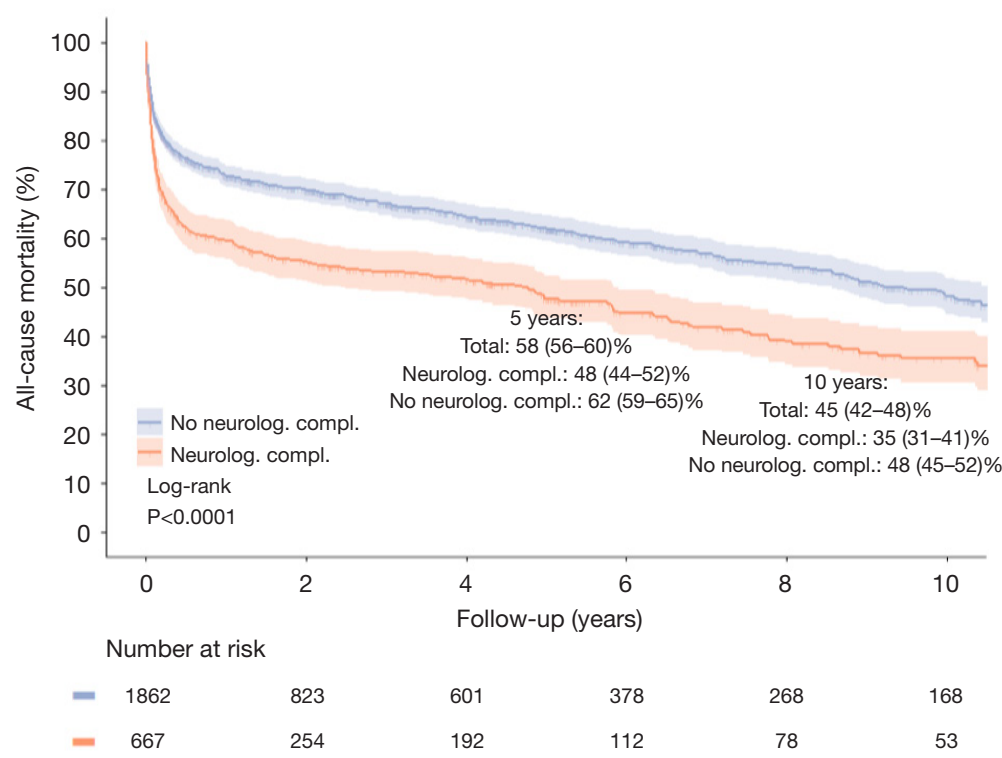

Figure 6 Kaplan-Meier curve for all-cause mortality in-house and in follow-up, stratified by preoperative neurological complications and annotated by 5 - and 10 -year survival with corresponding $95 \%$ confidence intervals.

mortality was $15.7 \%$. The most common cause of mortality was sepsis, followed by low cardiac output syndrome. Patients with preoperative neurological complications demonstrated significantly higher mortality rates at longterm follow-up compared to patients without neurological complications $(\mathrm{P}<0.0001$; Figure 6$)$.

\section{Discussion}

Neurological complications due to IE are common (9). We present one of the largest cohorts of IE patients and evaluate follow-up of patients presenting with and without neurological complications. The rate of neurological complications in our cohort was $26.8 \%$, which mirrors previously published studies $(2,9)$. We demonstrated that in-hospital mortality was higher in patients presenting with S. aureus than other organisms (3). Patients with vegetations greater than $1 \mathrm{~cm}$ on echocardiography had a significantly higher risk of embolization and further neurological complications than patients with smaller vegetations, which suggests surgical intervention at this cutoff length $(10,11)$. Additional risk factors in our cohort for cerebral embolization include endocarditis of the mitral or aortic valve, preoperatively initiated empiric antibiotic therapy, and identification of $S$. aureus pathogen.

García-Cabrera and colleagues found a higher mortality rate in a cohort of 121 patients with neurological complications due to IE, especially in the case of stroke (9). Their analysis 
also demonstrated embolized vegetations greater than $4 \mathrm{~mm}$ to be associated with ischemic cerebral lesions (9). Their mortality results are consistent with our cohort.

Neurological complications could be prevented in IE patients diagnosed and treated early (12). A significant proportion of patients suffering from IE need a multidisciplinary team of clinicians and surgeons to determine effective treatment for the patient. If neurological complications already exist, surgical management should be considered as a first option while balancing the risks inherent to anticoagulation during cardiopulmonary bypass (3). Thuny and colleagues demonstrated that surgical management is associated with improved neurological outcomes in IE patients presenting with silent cerebrovascular complications or transient ischemic attack (13). However, their observational study is prone to selection bias due to patients in the surgery group having indications for surgery, and the conservatively managed patients demonstrating significantly more comorbidities and lower Glasgow Coma Scale.

Presently, the decision of whether to intervene surgically after a stroke due to IE remains disputed. Recent studies indicate that early surgical treatment is a relatively safe and effective treatment option $(7,14)$. Studies have also demonstrated that valvular surgery can be safely performed after silent cerebrovascular complications or transient ischemic attack (13). In the case of intracranial haemorrhage, it is suggested to delay surgery for at least one month due to a worse neurological prognosis (15). Preoperative cerebral imaging is a fundamental part of standard diagnostic methods, particularly in the case of neurological complications. The higher sensitivity of MRI could make it a good diagnostic tool, however, it is not as readily available, takes longer to perform, and has higher costs.

Biological valves have recently become more common compared with mechanical valves. Our study also reflects this phenomenon as our institutional policy prefers implantation of biological valves in patients with IE. This is largely due to long-term anticoagulation not being required for patients with bioprosthetic valves. Anticoagulation does not have to be as strict as for mechanical valves - this is beneficial if haemorrhagic transformation of a cerebral infarct is observed post-operatively.

Misfeld and colleagues found that preoperative antibiotic treatment was a significant risk factor for cerebral embolism in their cohort (4). Preoperative antibiotic therapy in patients with left-sided endocarditis and large vegetations may lead to a fragmentation of the vegetations and consecutively lead to a higher incidence of systemic embolism (4).

The in-hospital mortality of $15.7 \%$ in our cohort was similar to previously published studies (16). In our cohort, the main reason for perioperative death was septic shock with consecutive multiorgan failure. Sepsis seems to be a main predictor for short- and long-term outcomes. These results are consistent with the study by Ruttmann and colleagues (17). Together, these results reflect the importance of early IE treatment with respect to long-term survival.

Unfortunately, patients with IE have a poor prognosis despite best medical care. The outcomes are considerably worse in patients presenting with neurological complications (9). In our cohort, patients with neurological complications showed a 5 -year survival of $48 \%$ compared with $62 \%$ in patients without neurological complications $(\mathrm{P}<0.0001)$. Ten-year survival was $35 \%$ versus $48 \%$ $(\mathrm{P}<0.0001)$, respectively. The results of our study reflected those of Thyny and colleagues; patients with preoperative neurological complications have higher long-term mortality compared to patients without neurological symptoms (14).

The indication for surgical intervention in infective endocarditis with or without neurological complications remains a multidisciplinary decision. However, several factors should be taken into account in deciding to operate. The clinical presentation is different and may include multiple symptoms or signs in the same patient, but focal signs predominate with ischaemic strokes most commonly diagnosed (3). S. aureus IE is more frequently associated with neurological complications compared with IE caused by other bacteria. The patient's hemodynamic profile can also affect the postoperative result. High dose inotropes correlate with poorer diagnosis. Imaging findings, including vegetation size and mobility, should also be taken into account (4). Many of the aforementioned characteristics found on patient presentation and examination were demonstrated to be associated with increased mortality, and should be accordingly taken into account when deciding on early surgical intervention for patients with IE.

\section{Conclusions}

In our cohort, we showed that long-term survival in patients with symptomatic neurological complications due to IE is associated with increased mortality compared to patients presenting without symptomatic neurological complications. Surgery remains a safe and effective treatment for IE patients with and without neurological complications. However, the 
short- and long-term outcomes are significantly poorer in those with neurological complications.

\section{Acknowledgments}

None.

\section{Footnote}

Conflicts of Interest: The authors have no conflicts of interest to declare.

\section{References}

1. Baddour LM, Wilson WR, Bayer AS, et al. Infective endocarditis in adults: diagnosis, antimicrobial therapy, and management of complications: a scientific statement for healthcare professionals from the American Heart Association. Circulation 2015;132:1435-86.

2. Murdoch DR, Corey GR, Hoen B, et al. Clinical presentation, etiology, and outcome of infective endocarditis in the 21st century: the International Collaboration on Endocarditis-Prospective Cohort Study. Arch Intern Med 2009;169:463-73.

3. Habib G, Lancellotti P, Antunes MJ, et al. 2015 ESC Guidelines for the management of infective endocarditis: The Task Force for the Management of Infective Endocarditis of the European Society of Cardiology (ESC). Endorsed by: European Association for Cardio-Thoracic Surgery (EACTS), the European Association of Nuclear Medicine (EANM). Eur Heart J 2015;36:3075-128.

4. Tornos P, Iung B, Permanyer-Miralda G, et al. Infective endocarditis in Europe: lessons from the Euro heart survey. Heart 2005;91:571-5.

5. Misfeld M, Girrbach F, Etz CD, et al. Surgery for infective endocarditis complicated by cerebral embolism: a consecutive series of 375 patients. J Thorac Cardiovasc Surg 2014;147:1837-44.

6. Piper C, Wiemer M, Schulte HD, et al. Stroke is not a contraindication for urgent valve replacement in acute infective endocarditis. J Heart Valve Dis 2001;10:703-11.
7. Thuny F, Beurtheret S, Mancini J, et al. The timing of surgery influences mortality and morbidity in adults with severe complicated infective endocarditis: a propensity analysis. Eur Heart J 2011;32:2027-33.

8. Li JS, Sexton DJ, Mick N, et al. Proposed modifications to the Duke criteria for the diagnosis of infective endocarditis. Clin Infect Dis 2000;30:633-8.

9. García-Cabrera E, Fernández-Hidalgo N, Almirante B, et al. Neurological complications of infective endocarditis: risk factors, outcome, and impact of cardiac surgery: a multicenter observational study. Circulation 2013;127:2272-84.

10. Iung B, Tubiana S, Klein I, et al. Determinants of cerebral lesions in endocarditis on systematic cerebral magnetic resonance imaging: a prospective study. Stroke 2013;44:3056-62.

11. Di Salvo G, Habib G, Pergola V, et al. Echocardiography predicts embolic events in infective endocarditis. J Am Coll Cardiol 2001;37:1069-76.

12. Dickerman SA, Abrutyn E, Barsic B, et al. The relationship between the initiation of antimicrobial therapy and the incidence of stroke in infective endocarditis: an analysis from the ICE Prospective Cohort Study (ICE-PCS). Am Heart J 2007;154:1086-94.

13. Thuny F, Avierinos JF, Tribouilloy C, et al. Impact of cerebrovascular complications on mortality and neurologic outcome during infective endocarditis: a prospective multicentre study. Eur Heart J 2007;28:1155-61.

14. Kang DH, Kim YJ, Kim SH, et al. Early surgery versus conventional treatment for infective endocarditis. N Engl J Med 2012;366:2466-73.

15. Yoshioka D, Sakaguchi T, Yamauchi T, et al. Impact of early surgical treatment on postoperative neurologic outcome for active infective endocarditis complicated by cerebral infarction. Ann Thorac Surg 2012;94:489-95.

16. Thuny F, Giorgi R, Habachi R, et al. Excess mortality and morbidity in patients surviving infective endocarditis. Am Heart J 2012;164:94-101.

17 Ruttmann E, Willeit J, Ulmer H, et al. Neurological outcome of septic cardioembolic stroke after infective endocarditis. Stroke 2006;37:2094-9.
Cite this article as: Jawad K, Kroeg G, Koziarz A, Lehmann S, Dieterlen M, Feder S, Garbade J, Rao V, Borger M, Misfeld M. Surgical options in infective valve endocarditis with neurological complications. Ann Cardiothorac Surg 2019;8(6):661-666. doi: 10.21037/acs.2019.10.04 